\title{
Clinical Efficacy of Antipsychotic Drugs in the Treatment of Schizophrenia
}

Felix-Martin Werner ${ }^{1,2^{*}}$ and Rafael Coveñas ${ }^{2}$

${ }^{1}$ Höhere Berufsfachschule für Altenpflege und Ergotherapie der Euro-Schulen Pößneck, Pößneck, Germany

${ }^{2}$ University of Salamanca, Institute of Neurosciences of Castilla y León (INCYL), Laboratory of Neuroanatomy of the Peptidergic Systems, Salamanca, Spain

*Corresponding author: Felix-Martin Werner, University of Salamanca, Instituto de Neurociencias de Castilla y León (INCYL) Laboratorio de Neuroanatomía de los Sistemas Peptidérgicos (Lab. 14) c/ Pintor Fernando Gallego, 1 37007-Salamanca, Spain, Tel: +34/923/29 44 00, Extn 1856, Fax: +34/923/29 45 49; E-mail: felixmwerner@versanet.de

Rec date: Oct 01, 2014, Acc date: Nov 09, 2014, Pub date: Nov 11, 2014

Copyright: (C) 2014 Werner F, et al. This is an open-access article distributed under the terms of the Creative Commons Attribution License, which permits unrestricted use, distribution, and reproduction in any medium, provided the original author and source are credited.

\begin{abstract}
We describe in schizophrenia the alterations of classical neurotransmitters and neuropeptides in the mesolimbic system, the hippocampus and the prefrontal cortex. The susceptibility genes, which encode dopamine hyperactivity and glutamate and GABA hyperactivity, are described. Using a neural network in the mesolimbic system, the coherence between the risk genes and the cellular mechanisms is derived. The therapeutic and adverse effects of the commonly prescribed second-generation antipsychotic drugs are pointed out. We suggest examining the risk genes in a large cohort of schizophrenic patients in order to have a tool to choose the appropriate antipsychotic drug. We recommend psychoeducation in the therapy of schizophrenic patients so that they can better deal with the schizophrenic symptoms and the adverse effects.
\end{abstract}

Keywords: Schizophrenia; Susceptibility gene; Antipsychotic drug; Dopamine; Serotonin; Psychoeducation

\section{Introduction}

Schizophrenia is a chronic mental illness which becomes manifest as an acute psychosis with positive schizophrenic symptoms such as paranoia, hallucinations and illusions after a prodromal phase with negative symptoms [1]. We mostly focus on the paranoid and hallucinatory form of schizophrenia. In this disease, some susceptibility genes have been discovered [2]. In the mesolimbic system and the hippocampus, dopamine hyperactivity via D2 receptors and serotonin hyperactivity via 5 -HT2A receptors occurs. In animal models of schizophrenia, schizophrenia-like behavior can be induced after administration of NMDA (N-methyl-D-aspartate) antagonists, which can be relieved by 5-HT2A antagonists, but not by D2 antagonists [3]. In this short communication, some susceptiblity genes and the coherence between the genetic localization and the cellular mechanisms are pointed out $[2,3]$. Schizophrenia is treated by administering second-generation antipsychotic drugs which exert a D2 and 5-HT2A antagonistic effect. The therapeutic and adverse effects of the commonly administered antipsychotic drugs are discussed [4].

\section{Alterations of Classical Neurotransmitters and Neuropeptides in Schizophrenia}

In schizophrenia, in the mesolimbic system and the hippocampus dopamine and serotonin hyperactivity and hypoactivity of presynaptic inhibitory neurotransmitters (GABA and glutamate) have been reported [5]. In the prefrontal cortex, D1 dopamine hyperactivity and M4 acetylcholine hypoactivity was found [5]. An antagonistic interaction between D1 dopaminergic and M4 muscarinic cholinergic neurons has also been described. The alterations of some neuropeptides, such as neurotensin, cholecystokinin and tachykinins will be also mentioned [5].

\section{Dopamine}

Dopamine hyperactivity, via D2 receptors, can be found in the mesolimbic system and the hippocampus and D1 dopamine hyperactivity in the prefrontal cortex (5). Dopamine hyperactivity can be associated with the susceptibility genes, for example the catechol-Omethyl transferase (COMT) and the monoamine oxidase (MAO) genes, which encode reduced dopamine degradation and thus lead to increased dopamine levels [6].

\section{Serotonin}

In schizophrenia, serotonin hyperactivity via 5 -HT2A receptors is partly encoded in polymophisms of the serotonin transporter gene [7]. Since in animal experiments schizophrenic-like behavior can be induced by NMDA antagonists, the following neural combination could be possible: a reduced presynaptic inhibition of 5-HT2A serotonergic neurons through glutaminergic neurons, via NMDA receptors, could as well contribute to serotonin hyperactivity [8].

\section{Gamma-aminobutyric Acid (GABA)}

A GABA hypofunction, via GABAA receptors, has been reported in the mesolimbic system and the hippocampus and it has been correlated with cognitive dysfunction [9]. In the above-mentioned brain areas, GABA hypoactivity is encoded in the glutamic acid decarboxylase (GAD) 67 gene $[2,10]$.

\section{Glutamate}

Glutamate, a mainly excitatory (postsynaptic excitatory) and partly presynaptic inhibitory neurotransmitter exerts its effect on NMDA receptors and on metabotropic glutaminergic receptors. Glutamate hypofunction, via NMDA receptors, is encoded in the dysregulin-1 and neuregulin-1 susceptibility genes [2]. In the mesolimbic sytem and the hippocampus, a reduced presynaptic inhibition via NMDA 
receptors of 5-HT2A serotonergic neurons could cause serotonin hyperactivity [5].

\section{Acetylcholine}

Acetylcholine is a postsynaptic excitatory neurotransmitter that exerts its function at muscarinic cholinergic and nicotinic cholinergic receptors. Agonists of both receptors could be of therapeutic value in the antipsychotic treatment. In schizophrenia, alpha7 and alpha4beta2 nicotinic cholinergic agonists improve cognitive functions [11]. M4 agonists could have antipsychotic properties. In schizophrenia, there is an antagonistic interaction between M4 muscarinic cholinergic and D1 dopaminergic neurons in the prefrontal cortex [12].

\section{Alterations of Neuropetides in Schizophrenia}

Among the altered neuropetides in schizophrenia, cholecystokinin, neurotensin and tachykinins will be mentioned.

Cholecystokinin (CCK) levels were found to be decreased in the striatum, the nucleus accumbens and in the frontal and temporal cortices [13]. The CCKA gene is associated with persistent auditory hallucinations in schizophrenia. It should be examined whether CCKA receptor agonists could be used as an additional therapy to improve these symptoms [14]. In schizophrenic patients, neurotensin levels were reduced in the mesolimbic system and the prefrontal cortex. Neurotensin agonists, which activate the NTS1 receptor and the neurotensin NT69L analogue, have been proved to show an antipsychotic effect [15]. Neurokinin A and B are of importance in the pathophysiology of schizophrenia. Neurokinin-3 receptor antagonists could have antipsychotic properties, because in the mesolimbic system they reduce the activity of D2 dopaminergic neurons [16].

\section{Susceptibility Genes in Schizophrenia and the Depending Pathophysiology}

The neuregulin-1, dysbindin-1, COMT, MAO and GAD 67 susceptibility genes will be described, and the coherence of these genes to the cellular mechanisms will be derived [3]. In the mesolimbic system, the neuregulin-1 and dysbindin-1 risk genes are linked to glutamate hypoactivity (2). Fatjó-Vilas et al. [17] examined ten dysbindin-1 single nucleotide polymorphisms (SNP) in 894 Caucasian individuals, namely 268 patients with a functional psychosis, 483 parents and 143 siblings and correlated the found SNP's with the patient's age at onset, the risk for psychosis and the familiar neurocognitive performance. These authors found different haplotypes of the dysbindin-1 gene, an haplotype (a 5-marker haplotype encompassing exons 2-4) was associated with an early onset of psychosis and other haplotypes were correlated with an adult-onset of schizophrenia [17]. The authors conclude from their findings that the haplotype of the dysbindin-1 gene is correlated with the form and severeness of schizophrenia [17]. The susceptibility genes COMT and monamine oxidase are linked with a decreased activity of these enzymes, catalyzing the breakdown of dopamine. Hence, in the mesolimbic system and the hippocampus dopamine hyperactivity occurs [5]. The GAD 67 gene is associated with a hypofunction of the presynaptic neurotransmitter GABA (3). In the mesolimbic system, the neural networks can be described as follows [18]: due to the neuregulin-1 and dysbindin-1 genes, glutaminergic neurons weakly inhibit presynaptically 5-HT2A serotonergic neurons which therefore have a high activity. The serotonergic neurons are connected to GABAergic neurons. Due to the GAD 67 gene, GABAergic neurons weakly inhibit presynaptically D2 dopaminergic neurons which exert a high activity. Dopamine hyperactivity is enhanced through the descreased activity of the COMT and MAO-B enzymes, which degrade dopamine. Tachykinin neurons, via NK3 receptors, strongly activate D2 dopaminergic neurons. In the ventral tegmental area, the dopaminergic neurons are connected to glutaminergic neurons. Dopamine and serotonin hyperactivity is still strengthened, because in the A10 cell group D2 dopaminergic and 5-HT2A serotonergic neurons activate each other [5]. GABAergic neurons in the mesolimbic system inhibit neurotensin neurons in the prefrontal cortex which weakly activate glutaminergic neurons via NTS1 receptors. GABAergic neurons in the ventral tegmental area also inhibit cholecystokinin neurons in the prefrontal cortex which weakly activate glutaminergic neurons via CCKA receptors. The glutaminergic neurons weakly inhibit serotonergic neurons in the VTA (ventral tegmental area) via NMDA receptors. Cannbinoid neurons strongly inhibit CCK neurons in the prefrontal cortex via CB1 receptors and have a hallucinogenic effect.

In the mesolimbic system, GABAergic neurons weakly inhibit, via GABAA receptors, D1 dopaminergic neurons in the prefrontal cortex (PFC), which strongly activate glutaminergic neurons. The latter neurons strongly inhibit via NMDA receptors M4 muscarinic cholinergic neurons, which weakly activate GABAergic neurons. These neurons weakly inhibit D1 dopaminergic neurons. The glutaminergic neurons in the PFC weakly inhibit via NMDA receptors serotonergic neurons in the mesolimbic system.

GABAergic neurons in the mesolimbic system weakly inhibit, via GABAA receptors, D2 dopaminergic neurons in the hippocampus which have a high activity. The D2 dopaminergic neurons are connected to glutaminergic neurons which weakly inhibit, via NMDA receptors, 5-HT2A serotoninergic neurons which have a high activity. The serotonergic neurons are connected to GABAergic neurons. Alpha4beta2 nAch neurons weakly activate GABAergic neurons. 5HT7 serotonergic neurons strongly activate 5-HT2A serotonergic neurons. The glutaminergic neurons in the hippocampus, via NMDA receptors, weakly inhibit the serotonergic neurons located in the mesolimbic system [18].

\section{Therapeutic and Adverse Effects of the commonly used Second-Generation Antipsychotic Drugs}

After schizophrenia is diagnosed, treatment with second-generation antipsychotic drugs (SGAs) is started. In this chapter, the therapeutic and adverse effects of the commonly used SGAs are described.

Risperidone is a D2 and 5-HT2A antagonist with a great affinity for the D2 receptor. Due to its mechanism of action, it induces extrapyramidal symptoms (EPS) more often than olanzapine, and hyperprolactinemia. It improves negative symptoms, for example social withdrawal, autism and depression, like other antipsychotic drugs such as ziprasidone. It causes metabolic side effects, but less often than olanzapine $[4,19,20]$. Olanzapine is a SGA with a greater affinity for the 5-HT2A receptor than risperidone. Olanzapine has a superior effect than other second-generation antipsychotic drugs to improve negative schizophrenic symptoms [19] and causes less often EPS than risperidone. In comparison to other SGAs, it causes metabolic side effects (glucose and cholesterol increases, weight gain) to a great extent $[4,21]$. Quetiapine is a SGA with a greater affinity for the 5-HT2A receptor than olanzapine; seldom causes EPS and it is a prolactin-sparing SGA. It has a therapeutic effect on positive and 
negative symptoms like risperidone [4]. Ziprasidone is a SGA with an additional antidepressant effect through a 5-HT1A agonistic effect. It improves positive and negative schizophrenic effects and has side effects, which are comparable to those induced by olanzapine [21]. Clozapine has a D3, D4 and 5-HT2A antagonistic effect and exerts the strongest antipsychotic effect. It seldom causes EPS and is a prolactinsparing antipsychotic drug. The occurrence of neutropenia, which may occur in $3 \%$ of the patients, must be controlled by a weakly blood cell count [4]. Aripiprazole has a different mechanism of action. It has a D2 partial agonism, a 5-HT2A antagonistic effect and a 5-HT1A agonistic effect. It has been shown to have a secure antipsychotic effect and seldom causes EPS and weight gain [19]. Up to now, there has not been chosen a way to select the appropriate antipsychotic drug [5].

\section{Conclusion}

In schizophrenic patients, we have described the alterations of classical neurotransmitters and neuropetides in the mesolimbic system, the hippocampus and the prefrontal cortex and we have also established the coherence between the susceptibility genes and the cellular mechanisms. Among the commonly prescribed secondgeneration antipsychotic drugs, risperidone has a greater affinity for the $\mathrm{D} 2$ receptor, and olanzapine and quetiapine have a greater affinity for the 5-HT2A receptor. In a large cohort of schizophrenic patients, the risk genes for the disease should be examined. It should be also examined if a specific antipsychotic drug has a better therapeutic effect when a certain susceptibility gene has been found; for example, it should be studied whether risperidone which shows a great affinity for the D2 receptor has a better therapeutic effect in patients with the COMT and MAO-B risk genes, i.e. the patients have an increased dopamine hyperactivity [5]. The combination of two antipsychotic drugs, for example risperidone and quetiapine can better treat schizophrenic symptoms; however the side effects must be considered [4]. After psychotic symptoms have improved, the dosage of the antipsychotic drug can be reduced, but a basic dose must be maintained, and the occurrence of side effects may occur less often. The patients should have a psychoeducation in order to deal with positive and negative symptoms and to recognize a recurrence of psychotic symptoms [4]. In clinical trials, it was shown that newer antipsychotic drugs with a different mechanism of action like aripiprazole have a safe therapeutic effect. In clinical trials, it should examined if recently developed antipsychotic drugs, like lurasidone and cariprazine, exert a long-lasting safe antipsychotic effect and if they can improve patients's quality of life [4].

\section{References}

1. Huber G, Gross G (1989) The concept of basic symptoms in schizophrenic and schizoaffective psychoses. Recenti Prog Med 80: 646-652.

2. Collier DA, Li T (2003) The genetics of schizophrenia: glutamate not dopamine? Eur J Pharmacol 480: 177-184.

3. Werner FM (2006) Schizophrenia: from the genetic localization to the cellular mechanism. Klin Neurol 37: 19-20.

4. Werner FM, Coveñas R (2014) Safety of antipsychotic drugs: focus on therapeutic and adverse effects. Expert Opin Drug Saf 13: 1031-1042.

5. Werner FM, Coveñas R (2013) Classical neurotransmitters and neuropeptides involved in major depression in a multi-neurotransmitter system: a focus on antidepressant drugs. Curr Med Chem 20: 4853-4858.
6. Gupta M, Kaur H, Jajodia A, Jain S, Satyamoorthy K, et al. (2011) Diverse facets of COMT: from a plausible predictive marker to a potential drug target for schizophrenia. Curr Mol Med 11: 732-743.

7. Aas M, Djurovic S, Athanasiu L, Steen NE, Agartz I, et al. (2012) Serotonin transporter gene polymorphism, childhood trauma, and cognition in patients with psychotic disorders. Schizophr Bull 38: 15-22.

8. Linck VM, Bessa MM, Herrmann AP, Iwu MM, Okunji CO, et al. (2012) 5-HT2A/C receptors mediate the antipsychotic-like effects of alstonine. Prog Neuropsychopharmacol Biol Psychiatry 36: 29-33.

9. Liu Y, Tang Y, Pu W, Zhang X, Zhao J (2011) Concentration change of DA, DOPAC, Glu and GABA in brain tissues in schizophrenia developmental model rats induced by MK-801. Zhong Nan Da Xue Xue Bao Yi Xue Ban 36: 712-719.

10. Gonzalez-Burgos G, Fish KN, Lewis DA (2011) GABA neuron alterations, cortical circuit dysfunction and cognitive deficits in schizophrenia. Neural Plast 2011: 723184.

11. Timmermann DB, Sandager-Nielsen K, Dyhring T, Smith M, Jacobsen AM, et al. (2012) Augmentation of cognitive function by NS9283, a stoichiometry-dependent positive allosteric modulator of $\hat{I} \pm 2$ - and $\hat{\mathrm{I}} \pm 4$ containing nicotinic acetylcholine receptors. Br J Pharmacol 167: 164-182.

12. Dencker D, Wörtwein G, Weikop P, Jeon J, Thomsen M, et al. (2011) Involvement of a subpopulation of neuronal M4 muscarinic acetylcholine receptors in the antipsychotic-like effects of the M1/M4 preferring muscarinic receptor agonist xanomeline. J Neurosci 31: 5905-5908.

13. Beinfeld MC, Garver DL (1991) Concentration of cholecystokinin in cerebrospinal fluid is decreased in psychosis: relationship to symptoms and drug response. Prog Neuropsychopharmacol Biol Psychiatry 15: 601-609.

14. Sanjuan J, Toirac I, González JC, Leal C, Moltó MD, et al. (2004) A possible association between the CCK-AR gene and persistent auditory hallucinations in schizophrenia. Eur Psychiatry 19: 349-353.

15. Li Z, Boules M, Williams K, Peris J, Richelson E (2010) The novel neurotensin analog NT69L blocks phencyclidine (PCP)-induced increases in locomotor activity and PCP-induced increases in monoamine and amino acids levels in the medial prefrontal cortex. Brain Res 1311: 28-36.

16. Malherbe P, Knoflach F, Hernandez MC, Hoffmann T, Schnider P, et al. (2011) Characterization of RO4583298 as a novel potent, dual antagonist with in vivo activity at tachykinin NKâ, and NK receptors. Br J Pharmacol 162: 929-946.

17. Fatjó-Vilas M, Papiol S, Estrada G, Bombín I, Peralta V, et al. (2011) Dysbindin-1 gene contributes differentially to early- and adult-onset forms of functional psychosis. Am J Med Genet B Neuropsychiatr Genet 156B: 322-333.

18. Werner FM (2014) Brain centers involved in schizophrenia. J Cytol Histol 5: 5.

19. Venkatesh PR, Kozielska M, Suleiman AA, Johnson M, Vermeulten A, et al. (2013) Pharmacokinetic-pharmacodynamic modeling of antipsychotic drugs in patients with schizophreniaPart I: The use of PANSS total score and clinical utility. Schizophr Res 146: 146-152.

20. Rummel-Kluge C, Komossa K, Schwarz S, Hunger H, Schmid F, et al. (2010) Head-to-head comparisons of metabolic side effects of second generation antipsychotics in the treatment of schizophrenia: a systematic review and meta-analysis. Schizophr Res 123: 225-233.

21. Thomas SP, Nandhra HS, Singh SP (2012) Pharmacologic treatment of first-episode schizophrenia: a review of the literature. Prim Care Companion CNS Disord 14.

This article was originally published in a special issue, entitled: "Histology and Histopathology", Edited by Borislav A. Alexiev, University of Maryland Medical Center, USA 